\title{
Advances in Unsupervised Learning Techniques Applied to Biosciences and Medicine
}

\author{
Anke Meyer-Baese, ${ }^{1}$ Sylvain Lespinats, ${ }^{2}$ Juan Manuel Gorriz Saez, ${ }^{3}$ and Olivier Bastien ${ }^{4}$ \\ ${ }^{1}$ Department of Scientific Computing, Florida State University, Tallahassee, FL 32306-4120, USA \\ ${ }^{2}$ Laboratoire des Systemes Solaires (L2S), Institut National de l'Energie Solaire (CEA/INES), BP 332, 73377 Le Bourget du Lac, France \\ ${ }^{3}$ Department of Signal Theory, Telematics and Communications, Facultad de Ciencias, Universidad de Granada Fuentenueva, s/n, \\ 18071 Granada, Spain \\ ${ }^{4}$ Laboratoire de Physiologie Cellulaire Végétale, UMR 5168 CEA-CNRS-INRA-Université Joseph Fourier, CEA Grenoble, 38054 \\ Grenoble Cedex 09, France
}

Correspondence should be addressed to Anke Meyer-Baese, ameyerbaese@fsu.edu

Received 29 August 2012; Accepted 29 August 2012

Copyright (๑) 2012 Anke Meyer-Baese et al. This is an open access article distributed under the Creative Commons Attribution License, which permits unrestricted use, distribution, and reproduction in any medium, provided the original work is properly cited.

\section{Introduction}

Artificial neural systems have been for the past thirty years a fascinating research topic with contributions from both theoreticians as well as from researchers implementing novel computational learning techniques into numerous application fields. Starting as an attempt to emulate human brain processing and cognition, supervised and unsupervised learning techniques as well as hybrid concepts have emerged for information visualization and discovery mining in high dimensional spaces. The properties of these spaces are very different from what we usually encounter in the more intuitive low dimensional spaces; the "curse of dimensionality," for example, often impacts the performance of data-mining tools. However, with the increasing demand of information and knowledge processing, and integration of information from heterogeneous sources into biomedical decision tools and resources for health care, innovative learning approaches become imperative. Especially, when facing a huge number of data descriptors as it is the case in almost all applications in life sciences.

The aim of this special issue is to present the current state of the art in the theory of unsupervised learning and applications by active experts researching in the vast area of biosciences and medicine.

\section{Novel Learning Algorithms}

The paper by X. Zhang et al. presents an indirect learning method that changes the synaptic weights by modulating spike-timing-dependent plasticity based on controlled input spike trains. V. Norris et al. propose a novel learning concept-competitive coherence-that appears to be relevant to a large group of biological systems and describe the differences to other existing content-addressable systems.

\section{Data Compression of Non-Gaussian Signals}

C. Plant et al.'s paper introduces a very general technique for evaluating ICA (Independent Component Analysis) results rooted in information-theoretic model selection and has potential applications to data compression.

\section{Applications in Brain Research}

A. Ortiz et al. proposes alternatives to MR (Magnetic Resonance) brain image segmentation algorithms based on selforganized maps and does not use any a priori information about the voxels in order to classify different tissue classes. R. Duan's and H. Man's paper proposes two novel unsupervised learning methods for analyzing functional magnetic resonance imaging (fMRI) data based on hidden Markov model (HMM). The HMM approach is focused on capturing the first-order statistical evolution among the samples of a voxel time series and not like conventional approaches to model the blood oxygen level-dependent (BOLD) response of a voxel as a function of time. 


\section{Applications in Mammography}

F. Steinbruecker et al.'s paper "Selection of spatiotemporal features in breast MRI (Magnetic Resonance Imaging) to differentiate between malignant and benign small lesions using computer-aided diagnosis" addresses the important aspect of motion compensation in breast MRI (Magnetic Resonance Imaging) and provides a new nonrigid technique for it. F. Steinbruecker et al.'s paper "Evaluation of a nano-rigid motion compensation technique based on spatiotemporal features for small lesion detection in breast MRI (Magnetic Resonance Imaging)" extracts spatiotemporal features for small lesions and evaluates their discriminative power in connection with motion compensation.

\section{Sleep Staging}

M. Langkvist et al.s paper addresses the important aspect of sleep stage classification. He proposes deep belief nets (DBN), an unsupervised learning paradigm, and shows how to apply it to sleep data in order to eliminate the use of handmade features.

\section{Hemodialysis}

The paper by T.-C. Lu et al. presents an entropy function to identify key features related to hemodialysis and based on these determined features a decision is made whether a patient needs hemodialysis.

In closing, many novel algorithmic techniques emerged for unsupervised learning and much more applications in biosciences and medicine are identified. Artificial neural network research has matured over the past decades and the power of unsupervised learning techniques is increasingly recognized. This special issue is brought to the reader with the hope to inform about the wealth of applications of unsupervised learning techniques and their indispensable role in biology and medicine.

\section{Acknowledgments}

We would like to thank all authors who have contributed to the special issue as well as all reviewers for their time and effort.

Anke Meyer-Baese Sylvain Lespinats Juan Manuel Gorriz Saez Olivier Bastien 

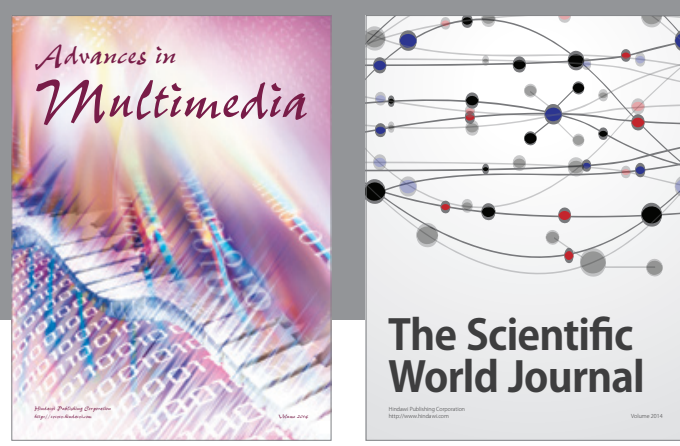

The Scientific World Journal
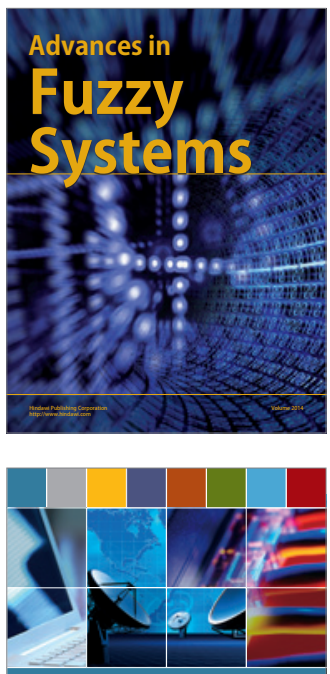

Computer Networks and Communications
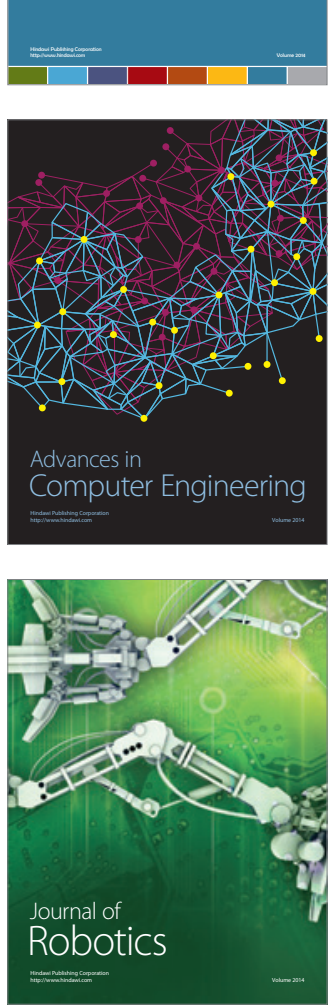
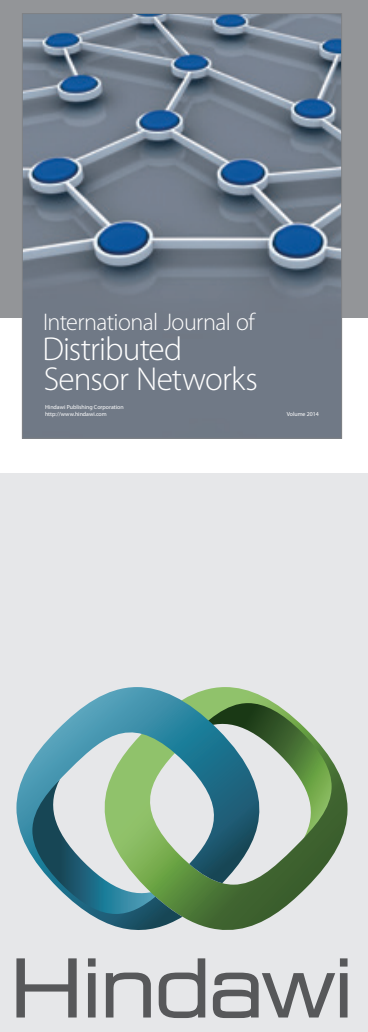

Submit your manuscripts at

http://www.hindawi.com
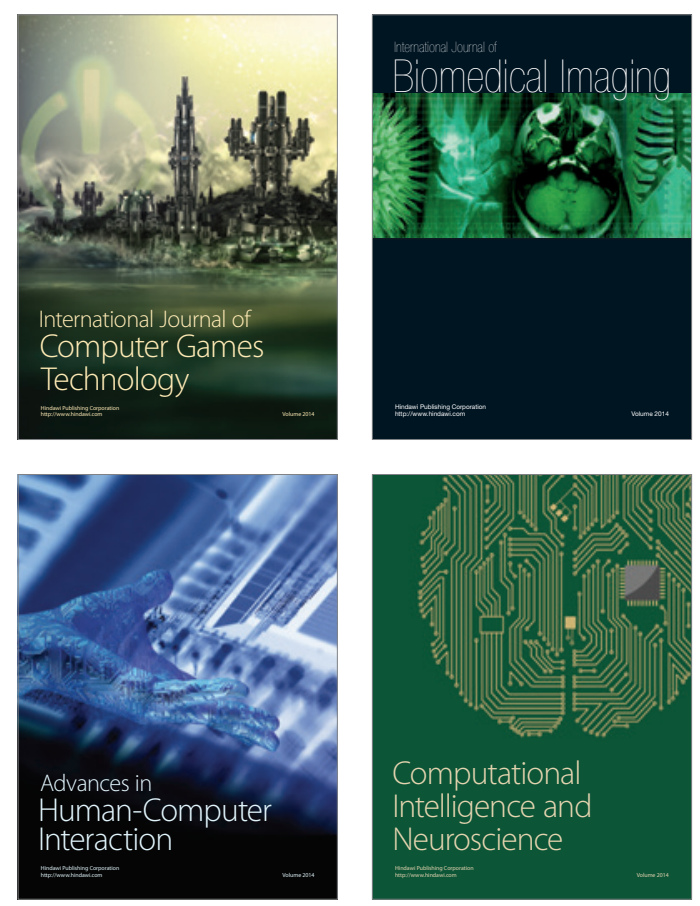
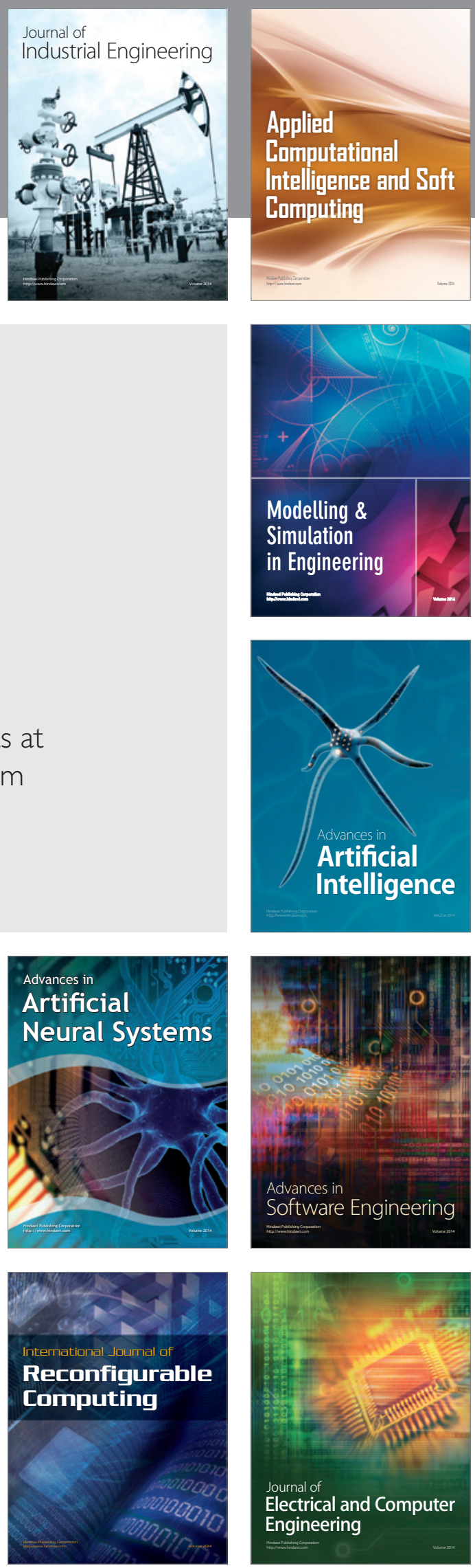